\title{
A novel hamster nonalcoholic steatohepatitis model induced by a high-fat and high-cholesterol diet
}

\author{
Yuta MIYAOKA ${ }^{1)}$, Denan JIN ${ }^{2}$, Keitaro TASHIRO ${ }^{1)}$, Shinsuke MASUBUCHI ${ }^{1)}$, Maiko OZEKI ${ }^{1)}$, \\ Fumitoshi HIROKAWA ${ }^{1)}$, Michihiro HAYASHI ${ }^{1}$, Shinji TAKAI ${ }^{2)}$, and Kazuhisa UCHIYAMA ${ }^{1)}$ \\ 1) Department of General and Gastroenterological Surgery, Osaka Medical College, 2-7 Daigaku-machi, Takatsuki- \\ shi, Osaka 569-8686, Japan \\ ${ }^{2)}$ Department of Innovative Medicine, Graduate School of Medicine, Osaka Medical College, 2-7 Daigaku-machi, \\ Takatsuki-shi, Osaka 569-8686, Japan
}

\begin{abstract}
Nonalcoholic steatohepatitis (NASH), in which there is steatosis and fibrosis in the liver, is linked to metabolic syndrome and progresses to hepatic cirrhosis. In this study, a novel hamster $\mathrm{NASH}$ model derived from metabolic syndrome was made using hamsters. Hamsters were fed a normal or a high-fat and high-cholesterol (HFC) diet for 12 weeks. Body weight and the ratio of liver weight to body weight were significantly greater in HFC diet-fed hamsters than in normal diet-fed hamsters. Triglyceride, low-density lipoprotein cholesterol, and glucose levels in blood were significantly increased in HFC diet-fed hamsters, and blood pressure also tended to be high, suggesting that the HFC diet-fed hamsters developed metabolic syndrome. Hepatic steatosis and fibrosis were observed in liver sections of HFC diet-fed hamsters, as in patients with $\mathrm{NASH}$, but they were not seen in normal diet-fed hamsters. Chymase generates angiotensin II and transforming growth factor (TGF)- $\beta$, both of which are related to hepatic steatosis and fibrosis, and a significant augmentation of chymase activity was observed in livers from HFC diet-fed hamsters. Both angiotensin II and TGF- $\beta$ were also significantly increased in livers of HFC diet-fed hamsters. Thus, HFC diet-fed hamsters might develop metabolic syndrome-derived NASH that clinically resembles that in NASH patients.
\end{abstract}

Key words: chymase, fibrosis, hamster, nonalcoholic steatohepatitis, steatosis

\section{Introduction}

Nonalcoholic fatty liver disease (NAFLD) has been recognized as the most common liver disease and typically presents as simple hepatic steatosis $[19,20]$. However, some cases of NAFLD progress from simple steatosis to nonalcoholic steatohepatitis (NASH), which mimics alcoholic hepatitis despite the absence of a history of drinking. NASH is a distinct entity characterized by severe steatosis and fibrosis of the liver [19, 20]. It is linked to metabolic syndrome by obesity, hyperlipidemia, hyperglycemia, and hypertension, and several clinical studies have investigated the treatment of NASH by focusing on the hyperlipidemia, diabetes, and hypertension $[3,8,13]$.

As a typical NASH model, rats fed a methionine- and choline-deficient (MCD) diet have been widely used as an experimental model. Although hepatic steatosis and fibrosis are observed after an MCD diet, excessive body weight loss is observed [5]. Therefore, the pathogenetic

(Received 6 October 2017 / Accepted 7 December 2017 / Published online in J-STAGE 1 January 2018)

Address corresponding: S. Takai, Department of Innovative Medicine, Graduate School of Medicine, Osaka Medical College, 2-7 Daigaku-machi, Takatsuki, Osaka 569-8686, Japan

c) $\$($ This is an open-access article distributed under the terms of the Creative Commons Attribution Non-Commercial No Derivatives (by-nc-nd) License <http://creativecommons.org/licenses/by-nc-nd/4.0/>. 
mechanism of MCD diet-induced NASH is different from that in NASH patients with metabolic syndrome. Recently, a rat NASH model produced in stroke-prone spontaneously hypertensive 5/Dmcr rats fed a high-fat and high-cholesterol (HFC) diet was reported $[6,11]$. This animal model developed manifestations of metabolic syndrome that were thought to resemble those of NASH patients $[6,11]$.

Increases of mast cell number were associated with the degree of human hepatic fibrosis in chronic liver diseases $[1,2,10]$. A mast cell stabilizer, tranilast, has been reported to prevent the development of hepatic fibrosis in a rat NASH model [21]. Although mast cells may be associated with NASH progression, they include several factors, such as histamine, cytokines, and protease, but which factors play crucial roles in the development and progression of NASH is unclear. Chymase is a serine protease contained in mast cells, and it converts angiotensin I to angiotensin II [16]. Angiotensin II blockade has been reported to prevent manifestations in patients with NASH $[3,22]$. The progression of NASH leads to liver cirrhosis, and augmentation of chymase and angiotensin II in the liver is closely related to the progression of fibrosis in patients with liver cirrhosis [7]. These findings suggest that chymase-dependent angiotensin II may contribute to the development and progression of NASH in patients. However, there are species differences in chymase-dependent angiotensin II-forming ability [16]. For example, human and hamster chymases convert angiotensin I to angiotensin II, but rat chymase does not [15]. To evaluate the role of chymase-dependent angiotensin II formation in NASH, such species differences need to be considered. In the present study, a novel NASH model derived from metabolic syndrome was produced using hamsters.

\section{Materials and Methods}

\section{Animal model}

Eight-week-old male hamsters $(\mathrm{n}=12)$ were obtained from Japan SLC (Shizuoka, Japan) and housed in a temperature-, humidity-, and light-controlled room. Normal and HFC diets were purchased from Funabashi Farm (Chiba, Japan), and the HFC diet contained 1\% cholesterol, $7 \%$ coconut oil, and 7\% corn oil. Normal and HFC groups were fed ad libitum with the normal and HFC diets for 12 weeks. All procedures involving animals were conducted in accordance with the "Guidelines for
Proper Conduct of Animal Experiments" (http://www. scj.go.jp/ja/info/kohyo-200-k16-2e.pdf).

\section{Systolic blood pressure and blood parameters}

Systolic blood pressure (SBP) was measured by the arm-cuff method (MK-2000ST, Muromachi Kikai Co., Ltd. Tokyo, Japan). After the animals were fasted for 18 $\mathrm{h}$, blood was obtained, and blood glucose levels were determined using a standard glucometer (Antosense II, Daikin Industries, Osaka, Japan).

Plasma was separated from the blood samples by centrifugation at 3,000 $\times \mathrm{g}$ for $15 \mathrm{~min}$ at $4^{\circ} \mathrm{C}$. Triglyceride and low-density lipoprotein (LDL) cholesterol levels were measured by SRL Inc. (Tokyo, Japan).

\section{Histological analysis}

Hepatic tissue specimens were fixed with Carnoy's fixative in $10 \%$ methanol overnight. Fixed liver tissues were embedded in paraffin and then cut at a thickness of $4 \mu \mathrm{m}$. The severity of hepatic histological changes was assessed using hematoxylin-eosin (HE) staining and Sirius red staining. Lipid droplets were observed in the HE-stained specimens, and the red area in the specimens stained with Sirius red was defined as the fibrotic region [11].

Mast cells were stained with $0.05 \%$ toluidine blue (Chroma-Gesellschaft, Stuttgart, Germany) at pH 4.8. To determine the distribution of chymase, immunohistochemical staining was performed using an anti-hamster chymase antibody (raised in rabbit by immunizing animals with SPYVPWINIVIKASS, which is a C-terminal peptide comprising amino acid residues 212 to 226 of hamster chymase) $[10,18]$. The sections were incubated for $24 \mathrm{~h}$ at $4^{\circ} \mathrm{C}$ with anti-chymase antibody and then reacted with the appropriate reagents from a streptavidinbiotin peroxidase kit (Dako LSAB kit, Dako North America, Inc., Carpinteria, CA, USA) and 3-amino9-ethylcarbazole, which was used for color development. The sections were counterstained with hematoxylin.

\section{Real-time polymerase chain reaction (RT-PCR)}

Liver total RNA was extracted using TRIzol reagent (Life Technologies, Rockville, MD, USA) and subsequently dissolved in RNase-free water (Takara Bio Inc., Kusatsu, Otsu, Japan) [11]. Total RNA (1 $\mu \mathrm{g})$ was transcribed into cDNA with SuperScript VILO (Invitrogen, Carlsbad, CA, USA) [11]. Then, mRNA levels were measured by RT-PCR on a Stratagene Mx3000P (Agi- 
lent Technologies, San Francisco, CA, USA) using TaqMan fluorogenic probes [11]. RT-PCR primers and probes for tumor necrosis factor (TNF)- $\alpha$, matrix metalloproteinase (MMP)-9, transforming growth factor (TGF)- $\beta$, collagen I, and $18 \mathrm{~S}$ ribosomal RNA (rRNA) were designed by Roche Diagnostics (Tokyo, Japan) [10]. The primers were as follows: 5'-CTGAGCCATCGTGCCAAT-3' (forward) and 5'-CCAGCTGGTTGTCTTTGAGA-3' (reverse) for TNF- $\alpha, 5^{\prime}$-CTTCGACGACGACGAGTTG-3' (forward) and 5'-TTGCGTTTCCAAAGTAAGTGG-3' (reverse) for MMP-9, 5'-GCTACCATGCCAACTTCTGC-3' (forward) and 5'-CCAGGACCTTGCTGTACTGTG-3' (reverse) for TGF- $\beta$, $5^{\prime}$-TGGACCTTGTTCACCTCTCTC- ${ }^{\prime}$ (forward) and 5'-CCCTGCTGGCAAAGATGTA-3' (reverse) for collagen I, and 5'-ATCCATTGGAGGGCAAGTC-3' (forward) and 5'-GCTCCCAAGATCCAACTACG-3' (reverse) for $18 \mathrm{~S}$ rRNA. The probes were as follows: $5^{\prime}$-CCTCCTGG- $3^{\prime}$ for TNF- $\alpha$, 5'-CTGGGCAA-3' for MMP-9, 5'-GAGCCTGG-3' for TGF- $\beta, 5^{\prime}$-CAGCAGGA-3' for collagen I, and 5'-CAGCAGCC-3' for $18 \mathrm{~S}$ rRNA. The mRNA levels of TNF- $\alpha$, MMP-9, TGF- $\beta$, and collagen I were normalized to that of 18S rRNA [11].

\section{Chymase activity and angiotensin II formation}

The liver was homogenized in $20 \mathrm{mM}$ Na-phosphate buffer ( $\mathrm{pH}$ 7.4) [7]. The homogenate was centrifuged at $15,000 \times \mathrm{g}$ for $30 \mathrm{~min}$. The supernatant was discarded, and the pellet was resuspended and homogenized in 10 $\mathrm{mM} \mathrm{Na}$-phosphate buffer ( $\mathrm{pH} 7.4$ ), containing $2 \mathrm{M} \mathrm{KCl}$ and $0.1 \%$ Nonidet P- 40 . The homogenate was centrifuged at $15,000 \times \mathrm{g}$ for $30 \mathrm{~min}$, and the supernatant was used for measurements of chymase activity [18]. Chymase activity in the liver extract was measured by incubating the tissue extracts with $5 \mathrm{mM}$ Suc-Ala-Ala-ProPhe-4-methylcoumaryl-7-amide (Peptide Institute Inc., Osaka, Japan) as the substrate [11]. One unit of chymase activity was defined as the amount of enzyme required to cleave $1 \mu \mathrm{M}$ of 7 -amino-4-methyl-coumarin/min.

Angiotensin II-forming activity was measured by incubating the tissue extract with $5 \mathrm{mM}$ angiotensin $\mathrm{I}$, and the formed angiotensin II was measured by an angiotensin II-measuring EIA kit (Bertin Pharma, Montigny le Bretonneux, France).

The protein concentration was assayed using BCA Protein Assay Reagents (Pierce, Rockford, IL, USA), with bovine serum albumin as the standard.

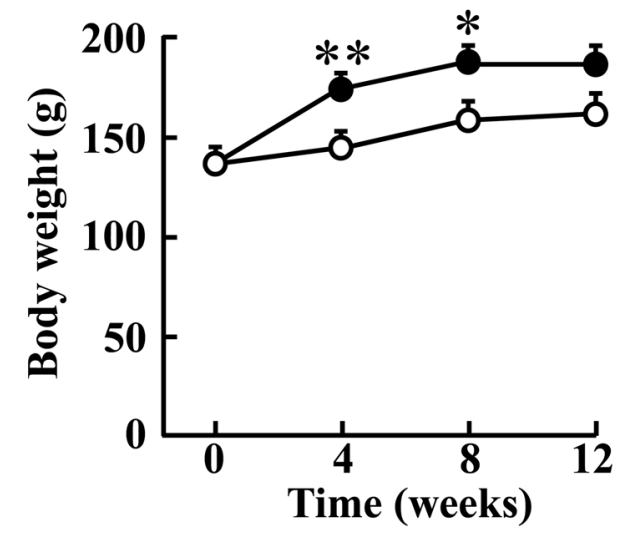

Fig. 1. Body weights in normal diet (open circle)and HFC diet (closed circle)-fed hamsters after $0,4,8$, and 12 weeks of the normal or HFC diet. Values represent the mean \pm SEM. $* P<0.05$ and $* * P<0.01$ vs. normal diet-fed hamsters.

\section{Western blotting}

Tissue extracts were analyzed in a Simple Western size-based capillary electrophoresis system (ProteinSimple Wes, ProteinSimple, San Jose, CA, USA). The size-separated proteins were probed with antibodies specific for hamster chymase (1:50), TGF- $\beta$ (1:50, Santa Cruz Biotechnology, Dallas, TX, USA), or GAPDH (1:100, R\&D Systems, Minneapolis, MN, USA), visualized using labeled secondary antibodies, and quantitated using the manufacturers' software. The intensities of the chymase and TGF- $\beta$ were normalized to the intensity of the GAPDH.

\section{Statistical analysis}

Data are expressed as means \pm SEM. Significant differences between mean values of the two groups were evaluated using Student's $t$-test for unpaired data. Values of $P<0.05$ were considered significant.

\section{Results}

\section{Body weight}

Body weight was significantly higher in HFC diet-fed hamsters than in normal diet-fed hamsters even after 4 weeks of the normal or HFC diet (Fig. 1). A significant difference was observed between the two groups at 8 weeks, but not at 12 weeks (Fig. 1). 
(A)

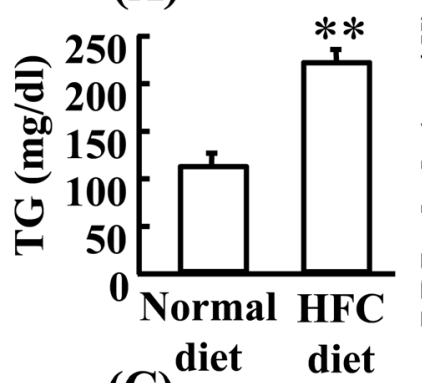

(C)

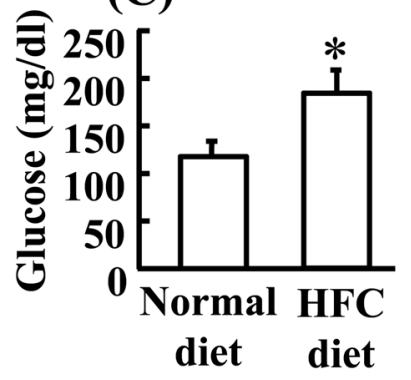

(B)

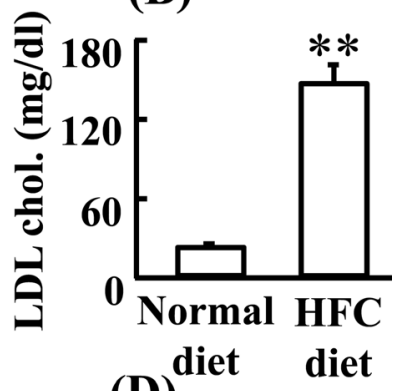

(D)

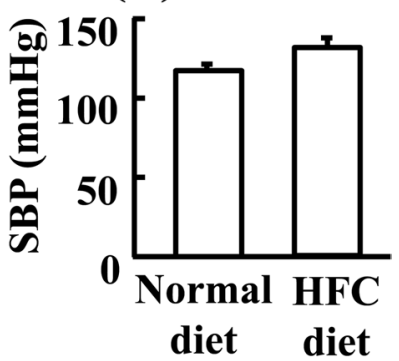

Fig. 2. Triglyceride (A), LDL cholesterol (B), and glucose (C) in blood from normal diet- and HFC diet-fed hamsters after 12 weeks of the normal or HFC diet. SBP in normal dietand HFC diet-fed hamsters after 12 weeks of the normal or HFC diet (D). Values represent the mean \pm SEM. $* P<0.05$ and $* * P<0.01$ vs. normal diet-fed hamsters.

\section{Blood parameters and $S B P$}

Twelve weeks after the normal or HFC diet, triglyceride and LDL cholesterol levels in plasma were significantly higher in HFC diet-fed hamsters than in normal diet-fed hamsters (Figs. 2A and B). A significant increase in blood glucose level was also observed in HFC diet-fed hamsters compared with normal diet-fed hamsters (Fig. $2 \mathrm{C})$. Although there was no significant difference between normal diet- and HFC diet-fed hamsters, SBP tended to be higher in HFC diet-fed hamsters (Fig. 2D).

\section{Whole liver and liver weight}

Typical photographs of whole livers from normal dietand HFC diet-fed hamsters after 12 weeks of each diet are shown in Fig. 3A. The color of the liver was paler in an HFC diet-fed hamster compared with a normal dietfed hamster, and the size was obviously larger in the HFC diet-fed hamsters than in the normal diet-fed hamsters (Fig. 3A).

Liver weight was higher in HFC diet-fed hamsters than in normal diet-fed hamsters (Fig. 3B). A significant increase in the ratio of liver weight to body weight was also observed in HFC diet-fed hamsters compared with

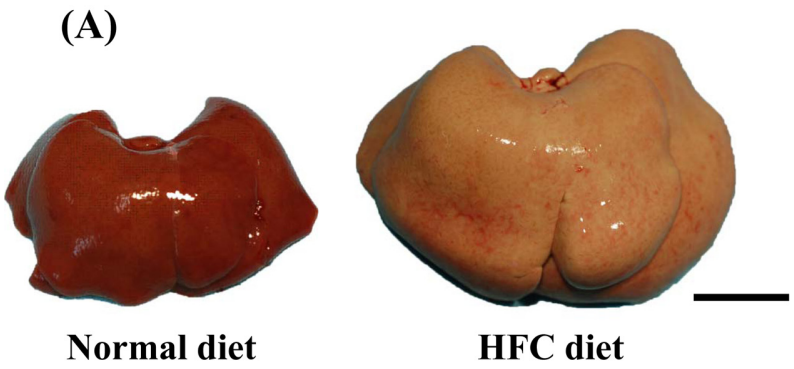

(B)

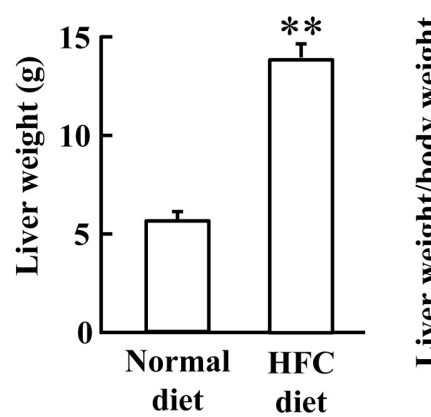

(C)

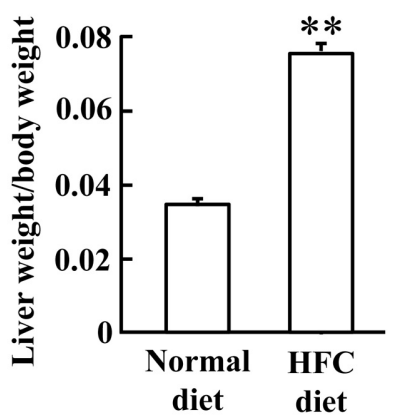

Fig. 3. Typical whole livers from normal diet- and HFC diet-fed hamsters after 12 weeks of the normal or HFC diet (A). The black bar represents $1 \mathrm{~cm}(\mathrm{~A})$. Liver weight (B) and ratio of liver weight to body weight (C) in normal diet- and HFC diet-fed hamsters after 12 weeks of the normal or HFC diet. Values represent the mean \pm SEM. $* * P<0.01$ vs. normal diet-fed hamsters.

normal diet-fed hamsters (Fig. 3C).

\section{Histological analysis of hepatic steatosis and fibrosis}

Typical photographs of HE- and Sirius red-stained liver sections from normal diet- and HFC diet-fed hamsters are shown in Fig. 4. Obvious lipid droplets were observed in HFC diet-fed hamsters, but they were not seen in normal diet-fed hamsters (Fig. 4A). MalloryDenk bodies, ballooning of hepatocytes, and infiltration of inflammatory cells were observed in the liver from HFC diet-fed hamsters (Fig. 4B), but they were not seen in normal diet-fed hamsters. Sirius red staining represents hepatic fibrosis, and many Sirius red-stained regions were observed in liver sections from HFC diet-fed hamsters, but the regions were obviously few in normal diet-fed hamsters (Fig. 4C). The ratios of fibrotic area to total liver area were $0.31 \pm 0.05 \%$ and $2.76 \pm 0.53 \%$ in normal diet- and HFC diet-fed hamsters, respectively, and the difference was significant $(P<0.01)$. 
(A)

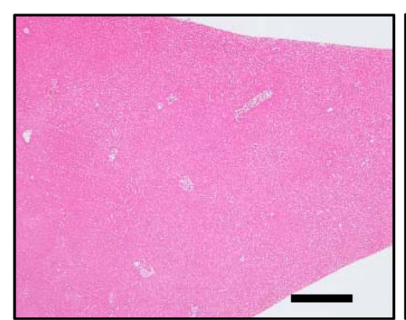

Normal diet

(B)

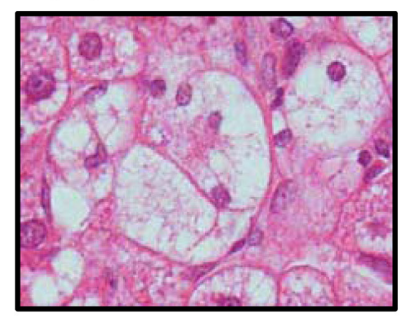

(C)

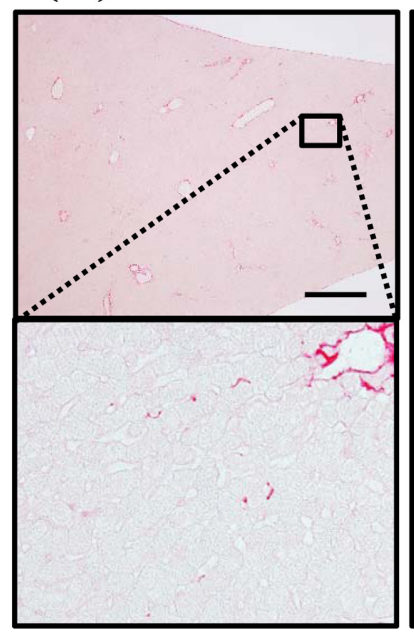

Normal diet

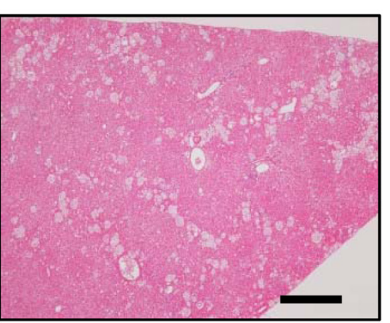

HFC diet
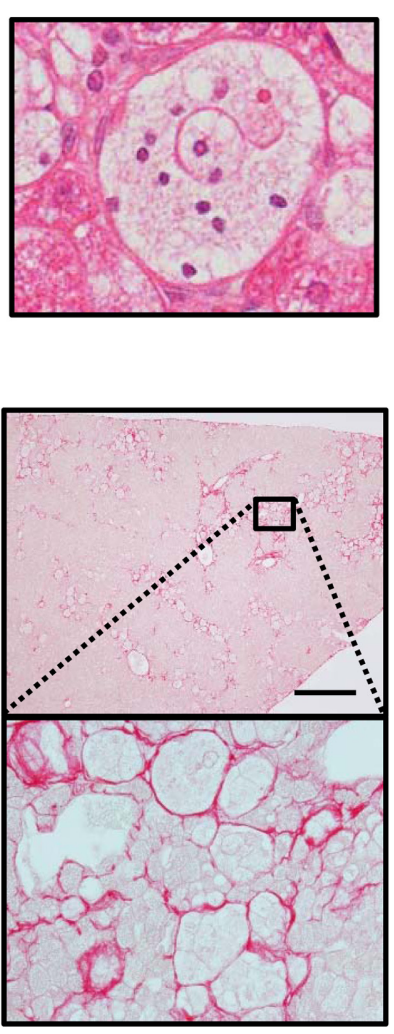

HFC diet

Fig. 4. Representative images of HE-stained liver sections from normal diet- and HFC diet-fed hamsters after 12 weeks of the normal or HFC diet (A). Typical images of MalloryDenk bodies (left side), ballooning of hepatocytes, and infiltration of inflammatory cells (right side) in HE-stained liver sections from HFC diet-fed hamsters after 12 weeks of the HFC diet (B). Representative images of Sirius redstained liver sections from normal diet- and HFC diet-fed hamsters after 12 weeks of the normal or HFC diet (C). Original magnification was 40x; scale bars represent 500 $\mu \mathrm{m}$ (A and $\mathrm{C}$, upper side).

Histological analysis of mast cells and chymase-positive cells

Typical photographs of toluidine blue-stained and

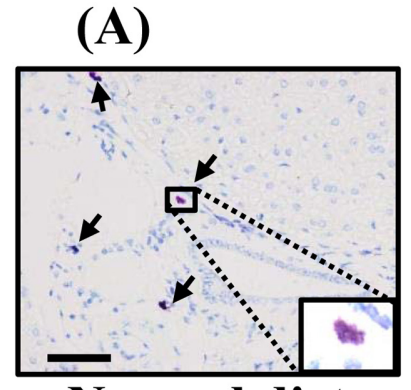

Normal diet

(B)

Normal diet

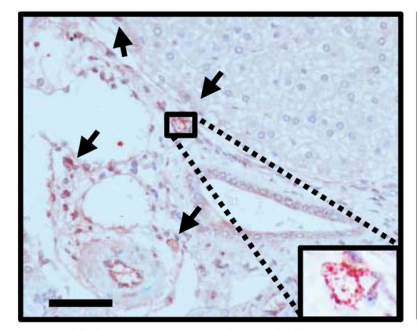

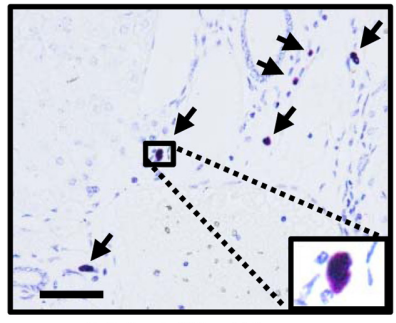

HFC diet

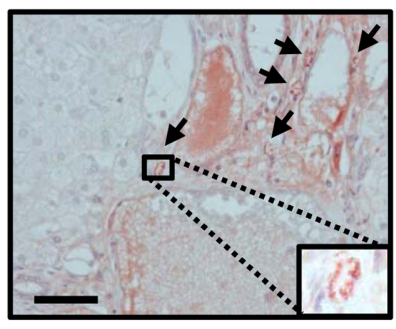

HFC diet
Fig. 5. Representative images of liver sections stained with toluidine blue (mast cells) (A) and immunostained with antichymase (chymase-positive cells) (B) from normal dietand HFC diet-fed hamsters after 12 weeks of the normal or HFC diet. Original magnification was 200x; scale bars represent $50 \mu \mathrm{m}$ (A and $\mathrm{B})$.

chymase-positive cells in liver sections from normal diet- and HFC diet-fed hamsters are shown in Fig. 5. Most chymase-positive cells were co-localized to toluidine blue-stained cells (Figs. 5A and B).

There were more toluidine blue-stained cells, which indicate mast cells, in liver sections from HFC diet-fed hamsters than normal diet-fed hamsters (Fig. 5A). Chymase-positive cells were also increased in HFC diet-fed hamsters compared with those in normal diet-fed hamsters (Fig. 5B). The mast cell numbers were $0.19 \pm 0.02$ and $0.25 \pm 0.04$ cells $/ \mathrm{mm}^{2}$ in normal diet- and HFC dietfed hamsters, respectively, and the chymase-positive cell numbers were $0.18 \pm 0.01$ and $0.23 \pm 0.04$ cells $/ \mathrm{mm}^{2}$ in normal diet- and HFC diet-fed hamsters, respectively. However, both mast cell and chymase-positive cell numbers were not significantly different between normal diet- and HFC diet-fed hamsters.

Hepatic mRNA levels of MMP-9, TNF- $\alpha, T G F-\beta$, and collagen I

Hepatic mRNA levels of TNF- $\alpha$, MMP-9, TGF- $\beta$, and collagen I were significantly higher in HFC diet-fed 
hamsters than in normal diet-fed hamsters (Figs. 6A-D).

Chymase activity and angiotensin II-formatting activity in liver extract

Chymase activity in liver extract was significantly
(A)

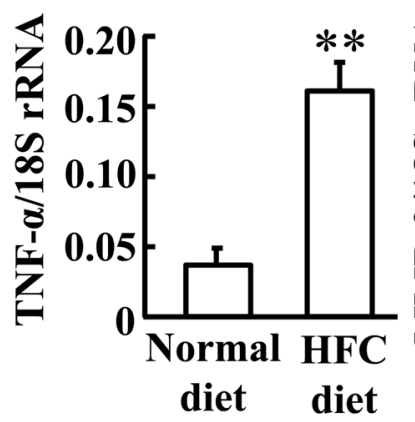

(C)

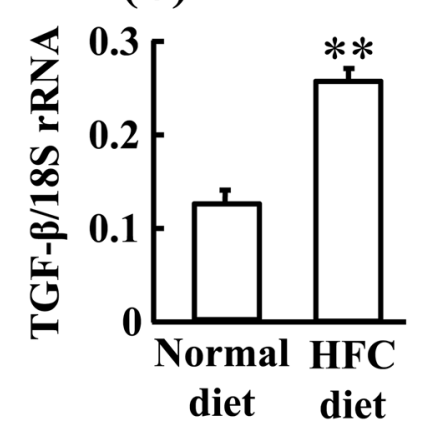

(B)

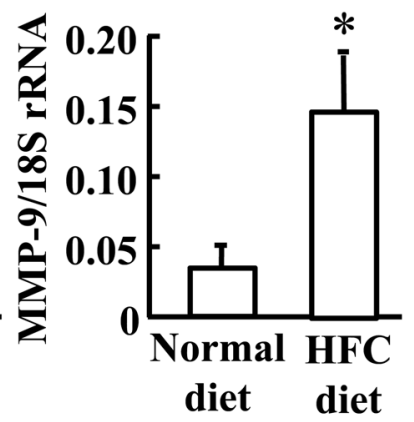

(D)

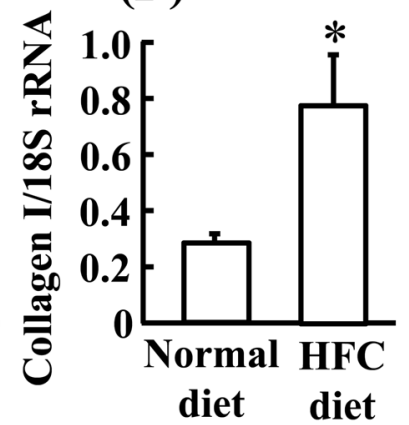

Fig. 6. Hepatic mRNA levels of TNF- $\alpha$, MMP-9, TGF- $\beta$, and collagen I. Hepatic mRNA levels of TNF- $\alpha$ (A), MMP-9 (B), TGF- $\beta(C)$, and collagen I (D) in liver extracts of normal diet- and HFC diet-fed hamsters after 12 weeks of the normal or HFC diet. Values represent the mean \pm SEM. $* P<0.05$ and $* * P<0.01$ vs. normal diet-fed hamsters. higher in HFC diet-fed hamsters than in normal diet-fed hamsters (Fig. 7A). A significant increase in angiotensin II-forming activity in liver extract was also observed in the HFC diet-fed hamsters compared with normal dietfed hamsters (Fig. 7B).

\section{Hepatic protein levels of chymase and TGF- $\beta$}

Chymase levels in liver extracts were significantly higher in HFC diet-fed hamsters than in normal diet-fed hamsters (Fig. 8A). A significant increase in the TGF- $\beta$ level in liver extracts was also observed in the HFC
(A)

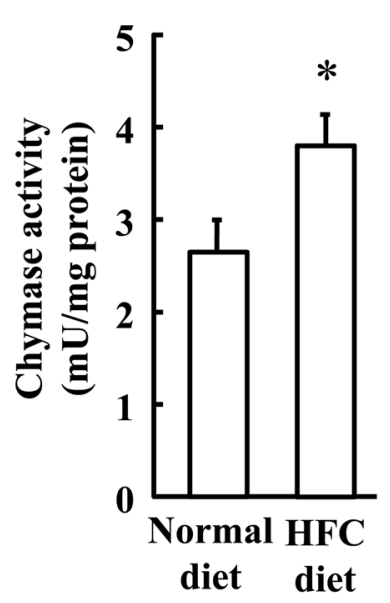

(B)

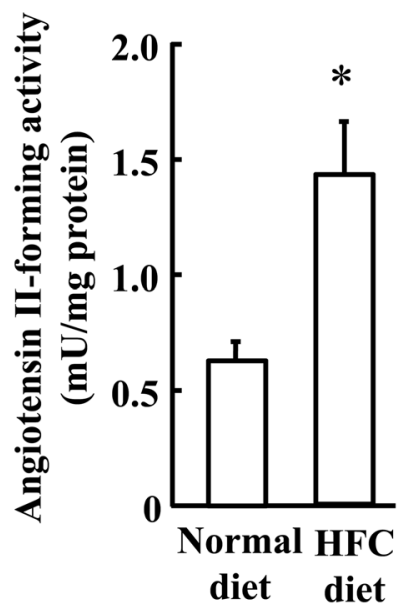

Fig. 7. Chymase activity (A) and angiotensin II-forming activity (B) in liver extract from normal diet- and HFC diet-fed hamsters after 12 weeks of the normal or HFC diet. Values represent the mean \pm SEM. $* P<0.05$ vs. normal diet-fed hamsters.
(A)

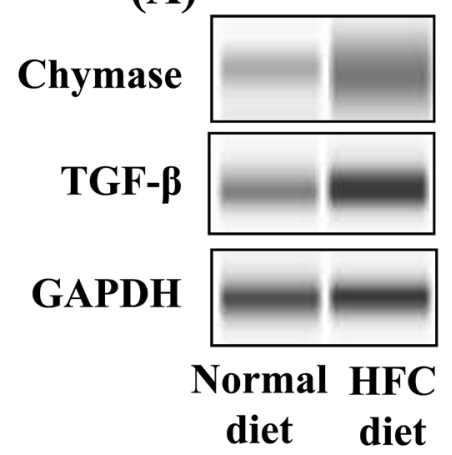

(B)

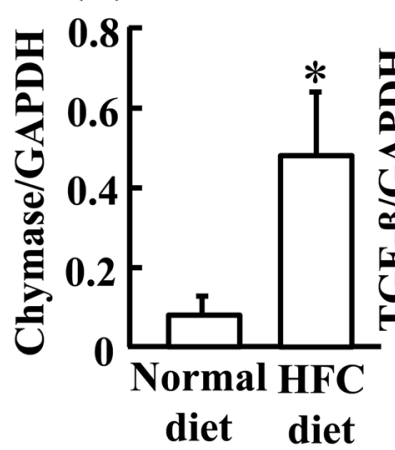

(C)

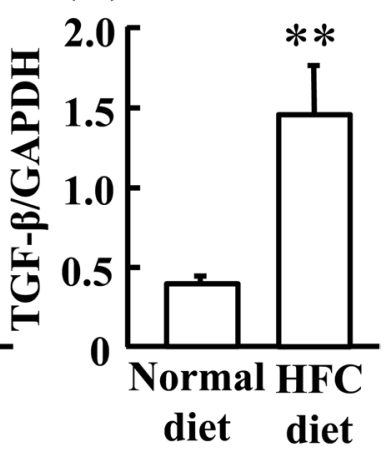

Fig. 8. Protein levels of chymase (A) and TGF- $\beta$ (B) in liver extract from normal diet- and HFC diet-fed hamsters after 12 weeks of the normal or HFC diet. Values represent the mean \pm SEM. $* P<0.05$ and $* * P<0.01$ vs. normal diet-fed hamsters. 
diet-fed hamsters compared with the normal diet-fed hamsters (Fig. 8B).

\section{Discussion}

Based on the results presented above, an HFC diet-fed model in hamsters may be used as a novel NASH model. Significant increases in body weight were observed in HFC diet-fed hamsters compared with normal diet-fed hamsters after 4 and 8 weeks of each diet, but no significant difference between normal diet- and HFC dietfed hamsters was observed at 12 weeks. The reason why the significant difference disappeared may have been the reduction in dietary intake in the HFC diet-fed hamsters. In normal diet-fed hamsters, dietary intake was gradually increased until 12 weeks, and the average weight of the diet consumed daily was $14.6 \mathrm{~g}$ per hamster. On the other hand, dietary intake gradually increased until 8 weeks in HFC diet-fed hamsters, like normal diet-fed hamsters, and no significant difference between normal diet- and HFC diet-fed hamsters was observed. However, dietary intake gradually decreased from 8 weeks to 12 weeks in HFC diet-fed hamsters, and the average weight of the diet consumed daily was $12.7 \mathrm{~g}$ per a hamster. The reason for the reduction may have been hepatic failure, although the actual reason was unclear. However, HFC diet-fed hamsters were clearly obese ip to the end of 8 weeks of the HFC diet. In the clinical setting, metabolic syndrome is diagnosed when two factors from among high LDL-cholesterol, high blood glucose, and high blood pressure are observed in addition to obesity. In the present study, both plasma triglyceride and LDL cholesterol levels were significantly higher in HFC diet-fed hamsters, and the blood glucose level was also significantly increased. Although SBP was not significantly increased after the HFC diet, SBP tended to be higher in HFC diet-fed hamsters. Therefore, this HFCdiet hamster model might be recognized as a metabolic syndrome model.

In patients with NASH, hepatic steatosis and fibrosis are observed in liver sections. In the present study, the color of the whole livers from HFC diet-fed hamsters were pale at 12 weeks, and their liver weights were significantly higher than those of normal diet-fed hamsters. In HE-stained liver sections, many lipid deposits were observed in livers from HFC diet-fed hamsters, and this pathological finding indicates hepatic steatosis. In Sirius red-stained liver sections, red-stained regions, which indicate fibrotic lesions, were clearly increased in HFC diet-fed hamsters, and this finding showed the progression of hepatic fibrosis. Mallory-Denk bodies, ballooning of hepatocytes, and infiltration of the inflammatory cells were also observed in HFC diet-fed hamsters, and this was similar to observations in human patients with NASH. Therefore, this HFC diet-fed hamster model showed manifestations of metabolic syndrome and also developed NASH; in other words, this model is a NASH model derived from metabolic syndrome.

In this study, hamsters were used as a metabolic syndrome-induced NASH model, although rats have been used as several obese-based NASH models [12, 14]. The study focused on chymase because we have demonstrated the significance of chymase in NASH pathogenesis $[10,18]$. Chymase catalyzes the formation of angiotensin II and TGF- $\beta$, both of which are related to the development and progression of hepatic fibrosis. Universally, chymases in humans, rats, hamsters, etc., change an inactive form, precursor TGF- $\beta$, to an active form, TGF- $\beta[9,17]$. On the other hand, species differences in angiotensin II-forming ability exist, and human and hamster chymases, but not rat chymase, convert angiotensin I to angiotensin II [16]. Therefore, like human chymase, the role of hamster chymase, including its angiotensin II-forming ability, needs to be studied. In the present study, significant increases in chymase and its activity were observed in livers from HFC diet-fed hamsters. Chymase-related factors, angiotensin II and TGF- $\beta$, were also increased in livers from HFC diet-fed hamsters. The augmentation of angiotensin II in addition to TGF- $\beta$ might be dependent on the increase in chymase, contributing to the development and progression of NASH in HFC diet-fed hamsters.

Chymase may be a useful target for prevention of NASH progression in patients. Angiotensin II blockade has been reported to prevent the development of symptoms in patients with NASH [3,22]. Some patients with NASH are known to develop hepatic cirrhosis, and there are significant correlations between angiotensin II and fibrotic degree, between chymase and fibrotic degree, and between angiotensin II and chymase in livers from patients with hepatic cirrhosis [7]. Chymase inhibitors have been shown to prevent the progression of cardiac fibrosis via the augmentation of angiotensin II and TGF- $\beta$ in animal models $[4,17]$. Although a chymase inhibitor, BAY 1142524 (Bayer), is currently in a phase II clinical trial (NCT02976467; www.clinicaltrials.gov) 
for the treatment of heart failure after myocardial infarction, chymase inhibitors cannot be generally used in the clinical setting. However, chymase inhibitors may be developed to prevent the progression of NASH.

In conclusion, a novel metabolic syndrome-induced NASH hamster model that was fed an HFC diet was proposed, and this model may become a useful NASH model for studying the role of angiotensin II-forming chymase, like human chymase.

\section{Conflict of Interest}

The authors declare that there are no conflicts of interest.

\section{Acknowledgments}

This study was partially supported by Grants-in-Aid for Scientific Research (C) of 17K10679 (FH), 17K09443 (MH), and 15K08251 (ST) from the Japan Society for the Promotion of Science.

\section{References}

1. Armbrust, T., Batusic, D., Ringe, B., and Ramadori, G. 1997. Mast cells distribution in human liver disease and experimental rat liver fibrosis. Indications for mast cell participation in development of liver fibrosis. J. Hepatol. 26: 10421054. [Medline] [CrossRef]

2. Farrell, D.J., Hines, J.E., Walls, A.F., Kelly, P.J., Bennett, M.K., and Burt, A.D. 1995. Intrahepatic mast cells in chronic liver diseases. Hepatology 22: 1175-1181. [Medline]

3. Georgescu, E.F., Ionescu, R., Niculescu, M., Mogoanta, L., and Vancica, L. 2009. Angiotensin-receptor blockers as therapy for mild-to-moderate hypertension-associated non-alcoholic steatohepatitis. World J. Gastroenterol. 15: 942-954. [Medline] [CrossRef]

4. Jin, D., Takai, S., Yamada, M., Sakaguchi, M., Kamoshita, K., Ishida, K., Sukenaga, Y., and Miyazaki, M. 2003. Impact of chymase inhibitor on cardiac function and survival after myocardial infarction. Cardiovasc. Res. 60: 413-420. [Medline] [CrossRef]

5. Kirsch, R., Clarkson, V., Shephard, E.G., Marais, D.A., Jaffer, M.A., Woodburne, V.E., Kirsch, R.E., and Hall, P.L. 2003. Rodent nutritional model of non-alcoholic steatohepatitis: species, strain and sex difference studies. J. Gastroenterol. Hepatol. 18: 1272-1282. [Medline] [CrossRef]

6. Kitamori, K., Naito, H., Tamada, H., Kobayashi, M., Miyazawa, D., Yasui, Y., Sonoda, K., Tsuchikura, S., Yasui, N., Ikeda, K., Moriya, T., Yamori, Y., and Nakajima, T. 2012. Development of novel rat model for high-fat and high-cholesterol diet-induced steatohepatitis and severe fibrosis progression in SHRSP5/Dmcr. Environ. Health Prev. Med. 17:
173-182. [Medline] [CrossRef]

7. Komeda, K., Jin, D., Takai, S., Hayashi, M., Takeshita, A., Shibayama, Y., Tanigawa, N., and Miyazaki, M. 2008. Significance of chymase-dependent angiotensin II formation in the progression of human liver fibrosis. Hepatol. Res. 38: 501-510. [Medline] [CrossRef]

8. Mahady, S.E., Webster, A.C., Walker, S., Sanyal, A., and George, J. 2011. The role of thiazolidinediones in non-alcoholic steatohepatitis - a systematic review and meta analysis. J. Hepatol. 55: 1383-1390. [Medline] [CrossRef]

9. Maruichi, M., Takai, S., Sugiyama, T., Ueki, M., Oku, H., Sakaguchi, M., Okamoto, Y., Muramatsu, M., Ikeda, T., and Miyazaki, M. 2004. Role of chymase on growth of cultured canine Tenon's capsule fibroblasts and scarring in a canine conjunctival flap model. Exp. Eye Res. 79: 111-118. [Medline] [CrossRef]

10. Masubuchi, S., Takai, S., Jin, D., Tashiro, K., Komeda, K., Li, Z.L., Otsuki, Y., Okamura, H., Hayashi, M., and Uchiyama, K. 2013. Chymase inhibitor ameliorates hepatic steatosis and fibrosis on established non-alcoholic steatohepatitis in hamsters fed a methionine- and choline-deficient diet. Hepatol. Res. 43: 970-978. [Medline] [CrossRef]

11. Miyaoka, Y., Jin, D., Tashiro, K., Komeda, K., Masubuchi, S., Hirokawa, F., Hayashi, M., Takai, S., and Uchiyama, K. 2017. Chymase inhibitor prevents the development and progression of non-alcoholic steatohepatitis in rats fed a high-fat and high-cholesterol diet. J. Pharmacol. Sci. 134: 139-146. [Medline] [CrossRef]

12. Ota, T., Takamura, T., Kurita, S., Matsuzawa, N., Kita, Y., Uno, M., Akahori, H., Misu, H., Sakurai, M., Zen, Y., Nakanuma, Y., and Kaneko, S. 2007. Insulin resistance accelerates a dietary rat model of nonalcoholic steatohepatitis. Gastroenterology 132: 282-293. [Medline] [CrossRef]

13. Park, H., Hasegawa, G., Shima, T., Fukui, M., Nakamura, N., Yamaguchi, K., Mitsuyoshi, H., Minami, M., Yasui, K., Itoh, Y., Yoshikawa, T., Kitawaki, J., Ohta, M., Obayashi, H., and Okanoue, T. 2010. The fatty acid composition of plasma cholesteryl esters and estimated desaturase activities in patients with nonalcoholic fatty liver disease and the effect of long-term ezetimibe therapy on these levels. Clin. Chim. Acta 411: 1735-1740. [Medline] [CrossRef]

14. Park, H.J., Lee, J.Y., Chung, M.Y., Park, Y.K., Bower, A.M., Koo, S.I., Giardina, C., and Bruno, R.S. 2012. Green tea extract suppresses NFאB activation and inflammatory responses in diet-induced obese rats with nonalcoholic steatohepatitis. J. Nutr. 142: 57-63. [Medline] [CrossRef]

15. Takai, S., Jin, D., Chen, H., Li, W., Yamamoto, H., Yamanishi, K., Miyazaki, M., Higashino, H., Yamanishi, H., and Okamura, H. 2014. Chymase inhibition improves vascular dysfunction and survival in stroke-prone spontaneously hypertensive rats. J. Hypertens. 32: 1637-1649. [Medline] [CrossRef]

16. Takai, S., Jin, D., and Miyazaki, M. 2012. Multiple mechanisms for the action of chymase inhibitors. J. Pharmacol. Sci. 118: 311-316. [Medline] [CrossRef]

17. Takai, S., Jin, D., Sakaguchi, M., Katayama, S., Muramatsu, M., Sakaguchi, M., Matsumura, E., Kim, S., and Miyazaki, M. 2003. A novel chymase inhibitor, 4-[1-([bis-(4-methyl- 
phenyl)-methyl]-carbamoyl)3-(2-ethoxy-benzyl)-4-oxoazetidine-2-yloxy]-benzoic acid (BCEAB), suppressed cardiac fibrosis in cardiomyopathic hamsters. J. Pharmacol. Exp. Ther. 305: 17-23. [Medline] [CrossRef]

18. Tashiro, K., Takai, S., Jin, D., Yamamoto, H., Komeda, K., Hayashi, M., Tanaka, K., Tanigawa, N., and Miyazaki, M. 2010. Chymase inhibitor prevents the nonalcoholic steatohepatitis in hamsters fed a methionine- and choline-deficient diet. Hepatol. Res. 40: 514-523. [Medline] [CrossRef]

19. Tilg, H. and Moschen, A.R. 2010. Evolution of inflammation in nonalcoholic fatty liver disease: the multiple parallel hits hypothesis. Hepatology 52: 1836-1846. [Medline] [CrossRef]

20. Tiniakos, D.G., Vos, M.B., and Brunt, E.M. 2010. Nonalco- holic fatty liver disease: pathology and pathogenesis. Annu. Rev. Pathol. 5: 145-171. [Medline] [CrossRef]

21. Uno, M., Kurita, S., Misu, H., Ando, H., Ota, T., Matsuzawa-Nagata, N., Kita, Y., Nabemoto, S., Akahori, H., Zen, Y., Nakanuma, Y., Kaneko, S., and Takamura, T. 2008. Tranilast, an antifibrogenic agent, ameliorates a dietary rat model of nonalcoholic steatohepatitis. Hepatology 48: 109-118. [Medline] [CrossRef]

22. Yokohama, S., Tokusashi, Y., Nakamura, K., Tamaki, Y., Okamoto, S., Okada, M., Aso, K., Hasegawa, T., Aoshima, M., Miyokawa, N., Haneda, M., and Yoneda, M. 2006. Inhibitory effect of angiotensin II receptor antagonist on hepatic stellate cell activation in non-alcoholic steatohepatitis. World J. Gastroenterol. 12: 322-326. [Medline] [CrossRef] 